\title{
QUESTIONS OF EXISTENCE AND UNIQUENESS FOR HYPERBOLIC EQUATIONS WITH DISCONTINUOUS COEFFICIENTS $\left({ }^{1}\right)$
}

\author{
BY \\ A. E. HURD AND D. H. SATTINGER
}

1. Introduction. In [3] I. M. Gelfand proposed the study of existence and uniqueness of solutions to the Cauchy problem for linear hyperbolic equations whose coefficients are possibly discontinuous. In this paper we present some sufficient conditions for the existence and uniqueness of weak solutions to such problems. We also present some simple examples of hyperbolic Cauchy problems for which weak solutions either do not exist or are not unique.

Our weak solutions are obtained as weak $L_{2}$ limits of solutions of the associated hyperbolic problem obtained by smoothing the coefficients and initial data. By placing appropriate conditions on the original equation, we can show that the strong solutions of the smoothed problem remain uniformly bounded in the $L_{2}$ norm on compact sets as the smoothing parameter goes to zero. By the diagonal method a subsequence of the family of smoothed solutions (indexed by the smoothing parameter) can be selected which converges weakly on compact sets.

The weak solutions obtained in this manner are in general not unique, but in some cases uniqueness can be obtained by placing an alternate condition on the coefficients. To illustrate this, we mention briefly the following example, which will be discussed in detail later. Consider the initial value problem

$$
u_{t}+(a(x, t) u)_{x}=0, \quad u(x, 0)=u_{0}(x) .
$$

By a weak solution of this problem we mean a locally integrable function $u(x, t)$ satisfying

$$
\int_{0}^{\infty} \int_{-\infty}^{\infty} u(x, t) \phi_{t}+a u \phi_{x} d x d t+\int_{-\infty}^{\infty} u(x, 0) \phi(x, 0) d x=0
$$

for any continuously differentiable function $\phi$ which vanishes for large $|x|+t$. Now consider the difference quotient

$$
Q(x, t, h)=(a(x+h, t)-a(x, t)) / h .
$$

If $Q(x, t, h)$ is bounded from below for all $h, t, x$, then a weak solution of (1) exists,

Presented to the Society, April 8, 1967; received by the editors January 3, 1967 and, in revised form, February 28, 1967 and April 12, 1967.

(1) This paper was sponsored in part by the U.S. Army Research Office (Durham) 31-124355, and NSF Grant GP-5279. Reproduction in whole or in part is permitted for any purpose of the United States Government. 
but may not be unique. On the other hand, by requiring that $Q$ be bounded from above, we shall guarantee uniqueness, but then (1) may fail to have a weak solution. By requiring that $Q$ be bounded from above and below we are in effect assuming that $a(x, t)$ is Lipschitz continuous in $x$; in this case, (1) has a unique weak solution. No assumptions need be made about the regularity of $a(x, t)$ in the $t$ variable.

Essentially the same result holds for first order symmetric hyperbolic systems in one space variable of the form

$$
U_{t}+(A U)_{x}+C=0
$$

where $U$ and $C$ are column vector valued functions, and $A$ is a matrix. Existence and uniqueness of weak solutions of (3) can be obtained by placing complementary jump conditions on the matrix $A$.

In the case of first order symmetric hyperbolic systems in several space variables,

$$
\left(A^{0} U\right)_{t}+\sum_{i=1}^{n} \frac{\partial}{\partial x_{i}}\left(A^{i} U\right)+B U+C=0,
$$

existence can be obtained by placing simple jump conditions on the matrix $A^{i}$ in the space variable $x_{i}$. No such simple condition, however, can be used to obtain uniqueness. In both cases no assumptions need be made on the regularity in the $t$ variable.

The situation is slightly different for second order equations of the form

$$
u_{t t}-\sum_{i, j=1}^{n} \frac{\partial}{\partial x_{i}}\left(a^{i j}(x, t) \frac{\partial u}{\partial x_{j}}\right)+\cdots=0 .
$$

In this case, questions of existence and uniqueness depend on the behavior of the leading coefficients $a^{i j}(x, t)$ in the time variable. We shall see in $\S 3$ that (5) has a weak solution if the quadratic form

$$
\sum_{i, j=1}^{n} a^{i j} v_{i} v_{j}
$$

satisfies certain jump conditions in $t$. By assuming complementary jump conditions we can guarantee uniqueness of the weak solution of (5), although then the weak solutions fail to exist in general. Finally, (5) has a unique weak solution if all the coefficients $a^{i j}(x, t)$ are Lipschitz continuous in $t$, while no regularity assumptions need be made concerning their behavior in the space variables $x_{1}, \ldots, x_{n}$.

The weak solutions we construct, both for second order equations and first order systems, will be shown to satisfy a classical energy inequality.

The jump condition (as in equation (2)) is similar to the entropy condition used by Oleinnik [5] in her investigations of the uniqueness of weak solutions of quasilinear conservation laws. Conditions similar to these jump conditions have been used by Conway [1] in the case of single equations in several space variables. 
N. N. Kuznecov [4] has demonstrated uniqueness of solutions of first order (nonsymmetric) hyperbolic systems in one space variable. He assumed that the coefficients were piecewise smooth and had simple jump discontinuities along smooth curves in the $x-t$ plane.

There are two approaches to hyperbolic equations with discontinuous coefficients. The first is the method of characteristics which involves considerations such as the intersection of characteristics and the Rankine-Hugoniot relations for shock discontinuities. This point of view is used in constructing our nonexistence examples, and it has also been developed extensively by Kuznecov [4]. The second approach is that of energy considerations, and it is this point of view that has been adopted in deriving the existence and uniqueness theorems in this paper. In this approach one tries to show by an approximation process that there is a finite energy integral associated with the solutions of the given hyperbolic equation with discontinuous coefficients. The jump conditions we apply to the coefficients allow them to have fairly bad behavior; in fact, the concept of characteristics need not make sense here at all.

2. Preliminaries. In this section we present two measure theoretic lemmas which will be used in the following discussions. We first introduce some notation that will be used throughout.

Let $E^{n+1}$ denote $(n+1)$-dimensional Euclidean space with points denoted by $x=\left(x_{0}, x_{1}, \ldots, x_{n}\right)$, and let $\mathscr{D}=\left\{x \in E^{n+1} ; x_{0} \geqq 0\right\}$. Let $H(t)$ be the hyperplane $x_{0}=t$. We denote the initial manifold $x_{0}=0$ by $H_{0}$. We shall sometimes treat the time variable $x_{0}=t$ separately and write points as $(\boldsymbol{x}, t)$, where $\boldsymbol{x}=\left(x_{1}, \ldots, x_{n}\right)$ is the space variable. The functions in our discussions will take on values in $E^{r}$, $r \geqq 1$; if $\langle$,$\rangle denotes the inner product in E^{r}$, we define $L^{2}(\mathscr{D})$ to be the set of all functions $u$ for which

$$
\int_{\mathscr{D}}\langle u, u\rangle d x<+\infty
$$

We denote by $L_{\text {loc }}^{2}(\mathscr{D})$ the set of all functions $u$ for which the above inequality is valid on compact subsets of $\mathscr{D}$. The dimension $r$ of the range space will usually be clear from the context.

We say that a sequence of functions $u_{n}(x)$ converges weakly to a function $u(x)$ on compact subsets of $\mathscr{D}$ if

$$
\lim _{n \rightarrow \infty} \int\left\langle u_{n}, \phi\right\rangle d x=\int\langle u, \phi\rangle d x
$$

for every function $\phi \in L_{\text {loc }}^{2}(\mathscr{D})$ with compact support in $\mathscr{D}$.

LEMMA 1. Let $\left\{u_{n}(\boldsymbol{x}, t)\right\}$ be a sequence of functions in $L_{\text {loc }}^{2}(\mathscr{D})$ which converges weakly on compact subsets of $\mathscr{D}$ to a function $u(x, t) \in L_{\mathrm{loc}}^{2}(\mathscr{D})$. Let $K(t)=K \cap H(t)$ 
where $K$ is some compact subset of $\mathscr{D}$. Suppose $\sigma(t)$ is some nonnegative function in $L_{\mathrm{loc}}^{2}([0, \infty))$ such that

$$
\int_{K(t)}\left\langle u_{n}(x, t), u_{n}(x, t)\right\rangle d x \leqq \sigma^{2}(t)
$$

Then for almost all $t$ we have

$$
\int_{K(t)}\langle u(x, t), u(x, t)\rangle d x \leqq \sigma^{2}(t) .
$$

Proof. Let $E$ be any compact measurable subset of $[0, \infty)$. By the weak convergence of $u_{n}$ we have

$$
\lim _{n \rightarrow \infty} \int_{E} \int_{K(t)}\left\langle u_{n}, u\right\rangle d x d t=\int_{E} \int_{K(t)}\langle u, u\rangle d x d t
$$

By Schwarz's inequality we see that

$$
\begin{aligned}
\left|\int_{E} \int_{K(t)}\left\langle u_{n}, u\right\rangle d x d t\right| & \leqq\left[\int_{E} \int_{K(t)}\left\langle u_{n}, u_{n}\right\rangle d x d t\right]^{1 / 2} \cdot\left[\int_{E} \int_{K(t)}\langle u, u\rangle d x d t\right]^{1 / 2} \\
& \leqq\left[\int_{E} \sigma^{2}(t) d t\right]^{1 / 2}\left[\int_{E} \int_{K(t)}\langle u, u\rangle d x d t\right]^{1 / 2}
\end{aligned}
$$

Therefore by (6) and (7) we have

$$
\left[\int_{E} \int_{K(t)}\langle u, u\rangle d x d t\right]^{1 / 2} \leqq\left[\int_{E} \sigma^{2}(t) d t\right]^{1 / 2} .
$$

Squaring both sides of the above inequality and letting $f^{2}(t)=\int_{K(t)}\langle u, u\rangle d x$, we get

$$
\int_{E} f^{2}(t) d t \leqq \int_{E} \sigma^{2}(t) d t
$$

Since this is true for arbitrary compact $E$, we have that $f(t) \leqq \sigma(t)$ a.e. Q.E.D.

LEMMA 2. Let $\Omega$ be a domain in $\mathscr{D}$ and let $w \in L^{2}(\Omega)$. Let $\left\{g_{n}(x)\right\}$ be a sequence of functions in $L^{2}(\Omega)$ whose norm is uniformly bounded:

$$
\int_{\Omega}\left\langle g_{n}, g_{n}\right\rangle d x \leqq G^{2}, \quad n=1,2, \ldots
$$

for some positive constant $G$. Let $A_{n}$ be a sequence of $r \times r$ matrices whose entries are uniformly bounded on $\Omega$ and converge to zero in $L^{2}(\Omega)$. Then

$$
\lim _{n \rightarrow \infty} \int\left\langle g_{n}, A_{n} w\right\rangle d x=0 .
$$

Proof. Lemma 2 is first proved for the case where $w$ is a characteristic function 
and then extended to the case where $w$ is a simple function by linearity. For arbitrary $w \in L^{2}(\Omega)$, one approximates $w$ by simple functions.

3. Symmetric hyperbolic systems. Consider in $\mathscr{D}$ the system

$$
L u=\sum_{i=0}^{n}\left(A^{i} u\right)_{i}+B u+C=0
$$

of partial differential equations for the vector function $u(x)=\left(u^{1}(x), \ldots, u^{r}(x)\right)$, where the matrices $A^{i}$ and $B$ and the vector $C$ are given functions of $x$, and the subscripts denote differentiation. We are concerned with establishing the existence, uniqueness, and continuous dependence of weak solutions of the Cauchy initial value problem for (8). Weak solutions are given by the following definition:

DEFINITION. $u$ is called a weak solution of (8) with the initial conditions $\psi(x)$ $\in L_{\mathrm{loc}}^{2}\left(H_{0}\right)$ if $u \in L_{\mathrm{loc}}^{2}(\mathscr{D})$ and

$$
\int_{\mathscr{D}} \sum_{i=0}^{n}\left[\left\langle A^{i} u, \phi_{i}\right\rangle-\langle B u, \phi\rangle-\langle C, \phi\rangle d x\right]+\int_{H_{0}}\left[\left\langle A^{0} \psi, \phi\right\rangle\right] d x=0
$$

for every vector function $\phi(x)$ which is continuously differentiable and which vanishes for large $|x|+t$.

We shall prove an existence theorem under the following conditions:

I. The elements of the matrices $A^{i}(0 \leqq i \leqq n), B$, and $C$ are in $L_{\text {loc }}^{2}(\mathscr{D})$.

II. The $r \times r$ matrices $A^{i}(0 \leqq i \leqq n)$ are symmetric.

III. There are constants $c_{0}>0$ and $c_{0}^{\prime}>0$ such that

$$
\left\langle A^{0}(x) u, u\right\rangle \leqq c_{0}\langle u, u\rangle
$$

for all $x \in \mathscr{D}$, and

$$
\left\langle A^{0}(x) u, u\right\rangle \geqq c_{0}^{\prime}\langle u, u\rangle
$$

for all $x \in H_{0}$, where $u$ is any vector.

IV. There is a constant $c_{1}>0$ such that, for all $x \in \mathscr{D}$ and all $i, 1 \leqq i \leqq n$,

$$
\left\langle A^{i}(x) u, u\right\rangle \leqq c_{1}\langle u, u\rangle
$$

for all vectors $u$.

V. There is a function $\mu \geqq 0$ in $L_{\text {loc }}^{1}([0, \infty))$ such that

$$
\langle B(x) u, u\rangle \geqq-\mu\left(x_{0}\right)\langle u, u\rangle
$$

for all vectors $u$ and all $x \in \mathscr{D}$.

VI. Let $\Delta x_{i}=x_{i}-\tilde{x}_{i}$ and correspondingly let

$$
\Delta_{i} A^{j}=A^{j}\left(x_{0}, \ldots, x_{i}, \ldots, x_{n}\right)-A^{j}\left(x_{0}, \ldots, \tilde{x}_{i}, \ldots, x_{n}\right) .
$$

Then there are functions $\mu_{i} \geqq 0 \in L_{\text {loc }}^{1}([0, \infty)), \mu_{0}$ being a constant, such that

$$
\left\langle\left(\Delta_{i} A^{i}(x) / \Delta x_{i}\right) u, u\right\rangle \geqq-\mu_{i}\left(x_{0}\right)\langle u, u\rangle
$$

for all vectors $u$ and all $x \in \mathscr{D}$. 
The existence theorem will be obtained via weak compactness arguments, using energy estimates for the solutions of problems obtained from (8) by the following smoothing process. Let $\omega_{k} \geqq 0(k=1,2, \ldots)$ be an infinitely differentiable function on $E^{n+1}$ with support contained in $|x| \leqq k^{-1}$ and satisfying

$$
\int_{E^{n+1}} \omega_{k}(x) d x=1
$$

For any $f \in L_{\text {loc }}^{2}(\mathscr{D})$ we define

$$
f_{k}(x)=\int_{E^{n+1}} \omega_{k}(x-y) f(y) d y .
$$

Then $f_{k}(k=1,2, \ldots)$ is infinitely differentiable in $\mathscr{D}$ and $f_{k}$ converges to $f$ in the $L_{2}$ norm on compact subsets of $\mathscr{D}$.

If $A(x)=\left(a^{i j}(x)\right)$ is a matrix with coefficients in $L_{\text {loc }}^{2}(\mathscr{D})$ we define $A_{k}(x)=\left(a_{k}^{i j}(x)\right)$. There is no difficulty in showing that conditions II, III, IV, and V for the matrices $A^{i}(0 \leqq i \leqq n)$ and $B$ carry over to the associated smoothed matrices $A_{k}^{i}$ and $B_{k}$. On the other hand, condition VI ensures that

$$
\left\langle\left(\partial A_{k}^{i} / \partial x_{i}\right) u, u\right\rangle \geqq-\mu_{i}\left(x_{0}\right)\langle u, u\rangle
$$

for any vector $u$, all $x \in \mathscr{D}$ and all $k$. To establish this fact consider the function

$$
F^{i}(x)=\left\langle\left[A^{i}(x)-\mu_{i}\left(x_{0}\right) x_{i} I\right] u, u\right\rangle
$$

where $u$ is a fixed vector, and all variables $x_{j}, j \neq i$, in $A^{i}(x)$ are regarded as fixed. Condition VI implies that $F^{i}(x)$ is monotone increasing as a function of $x_{i}$. The averaged function $F_{k}^{i}(x)$ is then also monotone increasing and is differentiable. Thus

$$
\partial F_{k}^{i} / \partial x_{i} \geqq 0
$$

and this condition immediately yields (11).

One can define a similar smoothing operation on the initial manifold $H_{0}$ which transforms $\psi \in L_{\text {loc }}^{2}\left(H_{0}\right)$ into infinitely differentiable functions $\psi_{k}(k=1,2, \ldots)$ which converge to $\psi$ in the $L_{2}$ norm of $H_{0}$ as $k \rightarrow \infty$.

We are now in a position to establish our first

THEOREM 1. Under assumptions I through $\mathrm{VI}\left({ }^{2}\right)$ there exists a weak solution $u \in L_{\mathrm{loc}}^{2}(\mathscr{D})$ of the Cauchy problem for (8) subject to the initial conditions $\psi \in L_{\mathrm{loc}}^{2}\left(H_{0}\right)$.

Proof. Consider the smoothed sequence of systems

$$
L_{k} u=\sum_{i=0}^{n}\left(A_{k}^{i} u\right)_{i}+B_{k} u+C_{k}=0
$$

together with the sequence of smoothed initial conditions

$$
u\left(0, x_{1}, \ldots, x_{n}\right)=\psi_{k}\left(x_{1}, \ldots, x_{n}\right) .
$$

$\left({ }^{2}\right)$ The bounds in V and VI actually need hold only on compact sets; a corresponding remark applies to condition (ii) in the case of second order equations. 
Conditions II and III for the system $L_{k}$ ensure that it is symmetric hyperbolic and so we can apply the existence and uniqueness theory for such systems [2]. In particular, we will apply it to the systems $L_{k}$ when they are restricted to the nested sequence of solid cones:

$$
\Gamma_{\tau}=\left\{x \in \mathscr{D}: \tau-x_{0} \geqq \frac{c_{0}}{c_{1}}\left(\frac{x_{1}^{2}+\cdots+x_{n}^{2}}{n}\right)^{1 / 2}, \tau=1,2, \ldots\right\} .
$$

From the existence theorem for equations with smooth coefficients we obtain, for each $\tau$, a sequence of continuously differentiable solutions $u_{\tau, k}(x)$ of $L_{k} u=0$ in $\Gamma_{\tau}$ which take on the values $\psi_{k}$ on $\Gamma_{\tau} \cap H_{0}$. The standard energy inequalities [2] show that

$$
E_{\tau, k}(t) \leqq \frac{c_{0}^{\prime}}{c_{0}}\left[1+\int_{0}^{t} k(s) \exp \left(\int_{s}^{t} k(u) d u\right) d s\right] E_{\tau, k}(0)
$$

where

$$
E_{\tau, k}(t)=\int_{H(t) \cap \Gamma_{\tau}}\left\langle u_{\tau, k}, u_{\tau, k}\right\rangle d \boldsymbol{x}
$$

and

$$
k(s)=\frac{1}{c_{0}} \max \left\{\max _{1 \leqq i \leqq n} \mu_{i}(s), \mu(s), 2 \int_{\Gamma_{\tau}}\langle C(s, x), C(s, x)\rangle d x_{1} \cdots d x_{n}\right\}
$$

Consequently

$$
\int_{\Gamma_{\tau}}\left\langle u_{\tau, k}, u_{\tau, k}\right\rangle d x \leqq Q \int_{\Gamma_{\tau} \cap H_{0}}\left\langle\psi_{k}, \psi_{k}\right\rangle d \boldsymbol{x}
$$

where $Q$ is some constant which does not depend on $k$.

We note in passing that the uniform lower bounds in V and VI are essential in deriving these estimates. The cones $\Gamma_{\tau}$ were chosen so that their sides are spacelike for all $k=1,2, \ldots$; hence the appropriate integrals over the sides of $\Gamma_{\tau}$ are positive allowing us to derive the energy inequality (15).

Clearly the right-hand side of $\left(15^{\prime}\right)$ is uniformly bounded above independently of $k$ and so by weak compactness we can select a subsequence of $\left\{u_{\tau, k}: k=1,2, \ldots\right\}$ which converges weakly to a function $u_{\tau}(x)$ on the cone $\Gamma_{\tau}$. Now for each $k$ the functions $u_{\tau, k}$ were weak solutions of $L_{k} u_{\tau, k}=0$ in $\Gamma_{\tau}$ in the sense that, if $\phi$ is a vector whose support in $D$ is contained in the interior of $\Gamma_{\tau}$, then

$$
\int_{\mathscr{D}}\left[\sum_{i=0}^{n}\left\langle A_{k}^{i} u_{\tau, k}, \phi_{i}\right\rangle-\left\langle B_{k} u_{\tau, k}, \phi\right\rangle-\left\langle C_{k}, \phi\right\rangle\right] d x+\int_{H_{0}}\left\langle A_{k}^{0} \psi_{k}, \phi\right\rangle d x=0
$$

Taking limits we see immediately that

$$
\int_{\mathscr{D}} \sum_{i=0}^{n}\left[\left\langle A^{i} u_{\imath}, \phi_{i}\right\rangle-\left\langle B u_{\tau}, \phi\right\rangle-\langle C, \phi\rangle\right] d x+\int_{H_{0}}\left\langle A^{0} \psi, \phi\right\rangle d \boldsymbol{x}=0 ;
$$

i.e., $u_{\tau}$ is a weak solution of $L u_{\tau}=0$ in $\Gamma_{\tau}$. By the diagonal method we can now find 
a subsequence of the functions $u_{\tau}$ which converges weakly on each cone $\Gamma_{\tau}$ to a function $u \in L_{\text {loc }}^{2}(\mathscr{D})$ which is the desired weak solution. Q.E.D.

Using Lemma 2 of $\$ 2$ we see immediately that the weak solution constructed in Theorem 1 satisfies a classical energy inequality, viz.,

$$
E_{\tau}(t) \leqq \frac{c_{0}^{\prime}}{c_{0}}\left[1+\int_{0}^{t} k(s) \exp \left(\int_{s}^{t} k(u) d u\right)\right] E_{\tau}(0)
$$

for all $\tau=1,2, \ldots$, where

$$
E_{\tau}(t)=\int_{\Gamma_{\tau} \cap H(t)}\langle u, u\rangle d x .
$$

Here, $u$ is our weak solution. This energy inequality does not establish uniqueness since there are weak solutions which do not satisfy this inequality, as we shall see later. The energy inequality, however, does establish continuous dependence of our weak solution on the initial data.

The condition VI which was used in the proof of the above existence theorem may at first seem somewhat artificial. To show that this assumption, or some alternative, is indispensible, we now present a simple Cauchy problem for a single first order equation in two variables which fails to satisfy Condition VI and for which a weak solution does not exist. Consider the equation

$$
u_{t}+[a(x) u]_{x}=0
$$

for the function $u(x, t)$ in the upper half-plane $D=\{(x, t): t \geqq 0\}$, where

$$
\begin{array}{rlrl}
a(x) & =0, & & x \leqq 0, \\
& =-1, & x>0,
\end{array}
$$

and $u(x, t)$ is subject to the initial conditions $u(x, 0) \equiv 1$. It is easy to see (by the uniqueness theorem for weak solutions which will be proved shortly) that in the regions $x<0$ and $x>0$ the weak solution $u(x, t)$ of (18), if it exists, must coincide with the solutions determined from the initial conditions in these respective regions by the method of characteristics. Hence we must have $u(x, t) \equiv 1$. But along any line of discontinuity $L$ the solution must satisfy the Rankine-Hugoniot jump conditions [2]

$$
[u] d x-[a u] d t=0
$$

where $[f]$ denotes the jump of the function $f$ along $L$. In the present case these conditions lead to the requirement that $u(x+0, t)=0$ for all $t>0$. Thus a weak solution in this case is over-determined by the initial data and in general cannot exist.

We shall now prove a uniqueness theorem for a restricted class of systems in one space variable. The method used is a variant of that of Holmgren [2].

Consider then the system

$$
M u=u_{0}+(A u)_{1}=0
$$


in $\mathscr{D}=\left\{\left(x_{0}, x_{1}\right): x_{0} \geqq 0\right\}$ for the vector function $u(x)=\left\{u^{1}(x), \ldots, u^{r}(x)\right)$. We suppose that $A$ satisfies

$I^{\prime}$. The elements of $A$ are in $L_{\text {loc }}^{\infty}(\mathscr{D})$.

II'. $A$ is a symmetric matrix.

IV $^{\prime}$. There is a constant $c>0$ such that for all $x \in \mathscr{D}$ and all vectors $u$,

$$
-c\langle u, u\rangle \leqq\langle A(x) u, u\rangle \leqq c\langle u, u\rangle \text {. }
$$

VI'. There is a function $\nu \in L_{\mathrm{loc}}^{1}([0, \infty))$ such that (using the notation of VI),

$$
\left\langle\left(\Delta_{1} A(x) / \Delta x_{1}\right) u, u\right\rangle \leqq v\left(x_{0}\right)\langle u, u\rangle
$$

for all vectors $u$ and all $x \in \mathscr{D}$.

From $\mathrm{VI}^{\prime}$ we see as before that the smoothed matrix $A_{k}$ associated with $A$ satisfies

$$
\left\langle\left(\partial A_{k} / \partial x\right) u, u\right\rangle \leqq v\left(x_{0}\right)\langle u, u\rangle
$$

We now present

THEOREM 2. If the above assumptions on $A$ are satisfied then any weak solution $u \in L_{\mathrm{loc}}^{2}(\mathscr{D})$ of $(20)$, which is zero on $H_{0}$, is identically zero.

Proof. The solution $u$ satisfies $\int_{\mathscr{D}}\left[\left\langle u, \phi_{0}\right\rangle+\left\langle A u, \phi_{1}\right\rangle\right] d x=0$ for any smooth vector function $\phi$. To show that $u(x) \equiv 0$, it suffices to show that $\int_{\mathscr{D}}\langle F(x), u(x)\rangle d x$ $=0$ for any infinitely differentiable vector function $F(x)$ which has compact support contained in $x_{0}>0$. We may choose $T$ large enough so that the support of $F$ is contained in $0<x_{0}<T$. Now let the functions $\phi^{k}(k=1,2, \ldots)$ be the solutions of the backward initial value problem $\phi_{t}^{k}+A_{k} \phi_{x}^{k}=F(x, t)$ on $0<t<T$ subject to $\phi^{k}(T, x)=0$. This can be done by solving the system

$$
-\tilde{\phi}_{t}^{k}+\tilde{A}_{k} \tilde{\phi}_{x}^{k}=\tilde{F}
$$

on $0<t<T, \tilde{\phi}^{k}(0, x)=0$ and then taking $\phi^{k}(x, t)=\tilde{\phi}^{k}(x, T-t)$. Here $\tilde{A}_{k}(x, t)$ $=A_{k}(x, T-t)$ and $\tilde{F}(x, t)=F(x, T-t)$. The function $\phi^{k}(x, t)$ so constructed vanishes for large $|x|+t$ and so is an admissible test function. Therefore

$$
\begin{aligned}
\int_{\mathscr{D}}\langle F, u\rangle d x & =\int_{\mathscr{D}}\left\langle\phi_{0}^{k}+A_{k} \phi_{x}^{k}, u\right\rangle d x \\
& =\int_{\mathscr{D}}\left[-\left\langle A u, \phi_{x}^{k}\right\rangle+\left\langle u, A_{k} \phi_{x}^{k}\right\rangle\right] d x \\
& =\int_{\mathscr{D}}\left\langle u,\left[A-A_{k}\right] \phi_{x}^{k}\right\rangle d x .
\end{aligned}
$$

Now it follows from I' that the elements of $A$ and $A_{k}$ are in $L_{\mathrm{loc}}^{\infty}(\mathscr{D})$, and also the elements of $A_{k}$ converge in mean square to those of $A$. If we assume for the moment that the functions $\phi_{x}^{k}$ are uniformly bounded in mean square as $k \rightarrow \infty$, then it follows immediately from these two facts and Lemma 2 that the right side of (22) can be made arbitrarily small, thus establishing the result. 
It remains to show that the functions $\phi_{x}^{k}$ are uniformly bounded in the $L_{2}$ norm as $k \rightarrow \infty$. Now

$$
\int_{-\infty}^{\infty} \int_{0}^{T}\left\langle\phi_{x}^{k}, \phi_{x}^{k}\right\rangle d x d t=\int_{-\infty}^{\infty} \int_{0}^{T}\left\langle\tilde{\phi}_{x}^{k}, \tilde{\phi}_{x}^{k}\right\rangle d x d t
$$

Furthermore, by differentiating the system for $\tilde{\phi}^{k}$ and letting $\tilde{W}^{k}=\tilde{\phi}_{x}^{k}$, we get the system

$$
\tilde{W}_{t}^{k}-\tilde{A}_{k} \tilde{W}_{x}^{k}-\left(\tilde{A}_{k}\right)_{x} \tilde{W}^{k}=-\tilde{F}_{x}
$$

Now the usual energy estimates show that the integrals

$$
\int_{-\infty}^{\infty} \int_{0}^{T}\left\langle\tilde{W}^{k}, \tilde{W}^{k}\right\rangle d x d t
$$

are uniformly bounded for $k=1,2, \ldots$ if

$$
\left\langle\left(\partial \tilde{A}_{k} / \partial x_{1}\right) u, u\right\rangle \leqq \nu\left(x_{0}\right)\langle u, u\rangle
$$

(along with the other assumptions made above). But the left hand side of (24) is the same as that of (21) and so we are through. Q.E.D.

One can extend the method to establish uniqueness results for equations in several space variables. To do so, however, one requires stronger assumptions in place of VI'. In all cases Lipschitz continuity in the space variables (uniformly on compact subsets) would suffice.

We will now present an example of nonuniqueness in a situation where assumption VI' does not hold. Consider the equation

$$
u_{t}+[b(x, t) u]_{x}=0
$$

in the upper half-plane $t \geqq 0$, where

$$
\begin{aligned}
b(x, t) & =0, & & x / t<1, \\
& =2, & & x / t>1,
\end{aligned}
$$

and $u(x, t)$ is subject to the boundary conditions $u(x, 0) \equiv 0$. Then using the method of characteristics we see that $u(x, t)$ is identically zero in the regions $x<0$ and $x / t>2$. However, the line $x / t=2$ is a characteristic, and solutions may have discontinuities across characteristics. Thus we may choose $u(x, t)$ to be any constant in the wedge $1<x / t<2$. Then $u$ will be determined in the region $0<x / t<1$ by the Rankine-Hugoniot conditions (the line $x / t=1$ being a line of discontinuity of the coefficient $b$ ). There will likewise be a jump in $u$ across $x=0$ but there are no restrictions here since this line is characteristic and $b(x, t)$ is continuous at $x=0$.

4. Existence and uniqueness of weak solutions of second order equations. Consider the second order hyperbolic equation

$$
u_{t t}-\sum_{i, j=1}^{n}\left(a^{i j} u_{i}\right)_{j}-b u_{t}-\sum_{i=1}^{n} c^{i} u_{i}-d u-e=0
$$


where the coefficients $a^{i j}, b, c^{i}, d$, and $e$ are bounded and measurable on compact subsets of $\mathscr{D}$ and satisfy the following additional conditions:

(i) There are constants $a$ and $a^{\prime}$ such that

$$
a^{\prime} \sum_{i=1}^{n} v_{i}^{2} \geqq \sum_{i, j}^{n} a^{i j}(x, t) v_{i} v_{j} \geqq a \sum_{i=1}^{n} v_{i}^{2}
$$

for any real numbers $v_{1}, \ldots, v_{n}$ and all points $(\boldsymbol{x}, t) \in \mathscr{D}$. We also assume that $a^{i j}=a^{j i}$.

(ii) There is a constant $A$ such that for all $t \neq t^{\prime}$, all $x \in E^{n}$, and any numbers $v_{1}, \ldots, v_{n}$

$$
\sum_{i, j=1}^{n} \frac{a^{i j}(x, t)-a^{i j}\left(x, t^{\prime}\right)}{t-t^{\prime}} v_{i} v_{j} \leqq A \sum_{i=1}^{n} v_{i}^{2}
$$

We extend $a^{i j}(x, t)$ to $t<0$ by setting $a^{i j}(x, t)=a^{i j}(x, 0)$ for $t<0$. As in $\S 3$ one may show by the properties of the smoothing kernel $\omega_{k}$ that condition (ii) implies that

$$
\sum_{i, j=1}^{n}\left[\frac{\partial}{\partial t} a_{k}^{i j}\right] v_{i} v_{j} \leqq A \sum_{i=1}^{n} v_{i}^{2}
$$

uniformly as $k \rightarrow \infty$.

We now define the Sobolev space $W_{\text {loc }}^{1,2}(\mathscr{D})$ as the set of all functions $u(x, t)$ $\in L_{\mathrm{loc}}^{2}(\mathscr{D})$ such that there are functions $u_{0}, u_{1}, \ldots, u_{n}$ in $L_{\text {loc }}^{2}(\mathscr{D})$ for which

$$
\begin{aligned}
& \iint_{\mathscr{D}} u \frac{\partial \phi}{\partial x_{i}} d x d t=-\iint_{\mathscr{D}} u_{i} \phi d x d t, \quad i=1, \ldots, n, \\
& \iint_{\mathscr{D}} u \frac{\partial \phi}{\partial t} d x d t=-\iint_{\mathscr{D}} u_{0} \phi d x d t-\int_{H_{0}} u(x, 0) \phi(x, 0) d x
\end{aligned}
$$

for any smooth function $\phi(x, t)$ which vanishes for large $|x|+t$. The functions $u_{i}$, $i=0, \ldots, n$, are called the distributional derivatives of $u$.

By a weak solution of $(25)$ we mean a function $u \in W_{\text {loc }}^{1.2}(\mathscr{D})$ with distributional derivatives $u_{t}, u_{1}, \ldots, u_{n}$ which satisfy

$$
\begin{aligned}
-\int_{-\infty}^{\infty} \phi(x, 0) u_{t}(x, 0) d x & +\iint_{\mathscr{D}}-u_{t} \phi_{t}+\sum_{i, j=1}^{n} a^{i j} u_{i} \phi_{j} d x d t \\
& -\iint_{\mathscr{D}} \phi(x, t)\left\{b u_{t}+\sum_{i=1}^{n} c^{i} u_{i}+d u+e\right\} d x d t=0
\end{aligned}
$$

for any $C^{2}$ function $\phi(x, t)$ which vanishes for large $|\boldsymbol{x}|+t$. The initial data $u(x, 0)$ and $u_{t}(x, 0)$ are assumed to belong to $L_{\text {loc }}^{2}\left(H_{0}\right)$.

We now prove the following theorem.

THEOREM 3. Under the assumptions (i) and (ii) above, the second order equation (25) has a weak solution in the sense (26). 
Proof. By the properties of the smoothing kernel we easily see that the inequalities (i) remain valid for the smoothed coefficients $a_{k}^{i j}$ as well. Thus the equation obtained from (25) by smoothing will be hyperbolic and we can apply the classical existence theory to obtain a strong solution $u^{k}$ with strong derivatives $u_{t}^{k}, u_{i}^{k}, i=1, \ldots, n$.

We now derive the classical energy inequality for the solution $u^{k}$ of the smoothed problem. We temporarily drop the superscript $k$ to avoid cumbersome notation. Multiply the equation for $u$ by $u_{t}$ and write the higher order terms as a divergence:

$$
\frac{1}{2}\left[\left(u_{t}\right)^{2}+\sum_{i, j=1}^{n} a^{i j} u_{i} u_{j}\right]_{t}-\sum_{i, j=1}^{n}\left(a^{i j} u_{i} u_{t}\right)_{j}=Q
$$

where

$$
Q=\frac{1}{2} \sum_{i, j} a_{t}^{i j} u_{i} u_{j}+b\left(u_{t}\right)^{2}+\sum_{i=1}^{n} c_{i} u_{i} u_{t}+d u u_{t}+e u_{t} .
$$

Here we have used the fact that $a^{i j}=a^{j i}$.

Let $P$ be the point $t=\tau, x_{1}=\cdots=x_{n}=0$ in the half space $\mathscr{D}$ and let $\Gamma_{\tau}$ be a cone with vertex $P$ and base on the hyperplane $H_{0}$. We shall specify $\Gamma_{\tau}$ exactly later. Let $\Gamma(t)=H(t) \cap \Gamma_{\tau}$. We define the energy integral

$$
E(t)=\frac{1}{2} \int_{\Gamma(t)}\left[\left(u_{t}\right)^{2}+\sum_{i, j=1}^{n} a^{i j} u_{i} u_{j}\right] d x .
$$

Now integrate the identity (27) over the region $R(t)$ bounded above by $\Gamma(t)$ $(t<\tau)$, on the sides by $\Gamma_{\imath}$, and below by $\Gamma(0)$. By Gauss' theorem we have

$$
\int_{\partial R}\left\{\frac{1}{2}\left[\left(u_{t}\right)^{2}+\sum_{i, j=1}^{n} a^{i j} u_{i} u_{j}\right] \xi_{0}-\sum_{i, j=1}^{n} a^{i j} u_{i} u_{t} \xi_{j}\right\} d S=\iint_{R(t)} Q(x, t) d x d t
$$

The vector $\left(\xi_{0}, \ldots, \xi_{n}\right)$ is the outward unit normal to the boundary of $R(t)$. The cone $\Gamma_{\tau}$ is chosen so that the integrand on the left side of (28) is nonnegative on the sides of $\Gamma_{\tau}$. We then have

$$
E(t)-E(0) \leqq \iint_{R(t)} Q(x, t) d x d t .
$$

We now want to estimate the right side of the above inequality in terms of $E(t)$. Note that $Q(x, t)$ depends on $u$ while $E(t)$ contains only first partial derivatives of $u$. We have, however,

$$
u(x, t)=\int_{0}^{t} u_{s} d s+u(x, 0)
$$

and by Schwarz's inequality,

$$
u^{2}(x, t) \leqq 2\left\{t \int_{0}^{t} u_{s}^{2} d s+u^{2}(x, 0)\right\}
$$


Integrating over $\Gamma(t)$ and using the fact that $\int_{\Gamma(t)} u_{t}^{2} d x \leqq 2 E(t)$, we obtain

$$
\begin{aligned}
\frac{1}{2} \int_{\Gamma(t)} u^{2}(x, t) d x & \leqq\left\{t \int_{0}^{t} \int_{\Gamma(t)} u_{s}^{2} d x d s+\int_{\Gamma(t)} u^{2}(x, 0) d x\right\} \\
& \leqq 2 t \int_{0}^{t} E(s) d s+\int_{\Gamma_{0}} u^{2}(x, 0) d x
\end{aligned}
$$

Here $\Gamma_{0}$ is some region in the initial manifold $H_{0}$ such that $\Gamma(t) \subset \Gamma_{0}$ for $0 \leqq t \leqq \tau$ and for all values of the smoothing parameter $k$. We are assured of the existence of such a region by assumption (i) which implies a uniform upper bound on the characteristic speeds of the smoothed equations; thus the domain of dependence of $P$ remains uniformly bounded as $k \rightarrow \infty$.

We can now estimate the integral of $Q(x, t)$ over the region $R(t)$. Let $B, C, D$ be positive constants such that $b(x, t) \leqq B,\left|C_{i}(x, t)\right| \leqq C$ for $i=1, \ldots, n$, and $|d(x, t)| \leqq D$. By the remark following condition (ii) and by the assumption of uniform ellipticity (ii) we have

$$
\begin{aligned}
\iint_{R(t)} Q(x, s) d x= & \int_{0}^{t} \int_{\Gamma(s)} Q(x, s) d x d s \\
\leqq & \int_{0}^{t} \int_{\Gamma(s)}\left\{\frac{A}{2 a} \sum_{i, j=1}^{n} a^{i j} u_{i} u_{j}+B u_{s}^{2}+\frac{C}{2}\left[\frac{1}{a} \sum_{i, j=1}^{n} a^{i j} u_{i} u_{j}+u_{s}^{2}\right]\right. \\
& \left.+\frac{D}{2}\left(u^{2}+u_{s}^{2}\right)+\frac{e^{2}+u_{s}^{2}}{2}\right\} d x d s .
\end{aligned}
$$

It is now clear that there is a constant $K$ depending on the positive numbers $a, A$, $B, C$, and $D$ such that

$$
\begin{aligned}
E(t)-E(0) \leqq & K \int_{0}^{t} E(s) d s+2 D \int_{0}^{t} s \int_{0}^{s} E\left(s^{\prime}\right) d s^{\prime} d s \\
& +\iint_{R(t)} e^{2}(x, s) d x d s+\int_{\Gamma_{0}} u^{2}(x, 0) d x .
\end{aligned}
$$

The constant $K$ does not depend on the smoothing parameter $k$ since the constants $a, A, B, C$, and $D$ are independent of $k$.

Letting

$$
f(t)=\iint_{R(t)} e^{2}(x, s) d x d s+\int_{\Gamma_{0}} u^{2}(x, 0) d x
$$

and integrating the iterated integral by parts we get

$$
E(t)-E(0) \leqq \int_{0}^{t}\left[K+D\left(t^{2}-s^{2}\right)\right] E(s) d s+f(t) .
$$

By assumption (i) the maximum characteristic speeds of the smoothed equations 
remain uniformly bounded as $k \rightarrow \infty$; hence $R(t)$ and therefore $f(t)$ remain uniformly bounded as $k \rightarrow \infty$. The above integral inequality implies that $E(t)$ remains bounded on $0 \leqq t \leqq \tau$ as $k \rightarrow \infty$.

The procedure for obtaining a global weak solution is essentially the same as in the case of first order symmetric systems. From (29) it is easily seen that the integrals

$$
\iint_{\Gamma_{\mathfrak{\tau}}}\left|u^{k}(\boldsymbol{x}, t)\right|^{2} d \boldsymbol{x} d t
$$

are uniformly bounded as $k \rightarrow \infty$. Letting $\tau=1,2, \ldots$ we construct by the diagonal method a subsequence of solutions $u^{\tau}$ such that the functions $u^{\tau}$ and $\left(u^{\tau}\right)_{i}, i=0, \ldots, n$ converge weakly on the cones $\Gamma_{\tau}$. It is easily seen that the weak limits thus obtained form a function $u \in W_{\text {loc }}^{1,2}(\mathscr{D})$ with weak derivatives $u_{t}, u_{1}, \ldots, u_{n}$ which together form a weak solution of (25). Q.E.D.

In the same manner as in the case of first order systems we can show, using Lemma 1 , that the weak solutions obtained satisfy an energy inequality.

We remark that if condition (ii) is not satisfied, then equation (25) may not have a weak solution. For example, consider the second order hyperbolic equation

$$
u_{t t}-\left(c^{2}(x, t) u_{x}\right)_{x}=0, \quad u(x, 0)=f(x) ; \quad u_{t}(x, 0)=g(x)
$$

where

$$
\begin{aligned}
c(x, t) & =a_{1}, & & x / t<1, \\
& =a_{2}, & & x / t>1,
\end{aligned}
$$

with $0<a_{2}<1<a_{1}$. Thus characteristics of the same type meet along the line $x=t$.

From the uniqueness theorem, which will be proved subsequently, it follows that in the region $x / t>1$ the weak solution must agree with the strong solution since the coefficient $c(x, t)$ is differentiable there. Continuity of the weak solution across the line $x=t$, (denoted by $L$ ) then prescribes $u(x, t)$ along the left side of $x=t$ (denoted by $\left.L^{-}\right)$. Denoting values along $L^{-}\left(L^{+}\right)$by $u^{-}\left(u^{+}\right)$we have $u^{-}=u^{+}$, hence $u_{t}^{-}+u_{x}^{-}=u_{t}^{+}+u_{x}^{+}$. The Rankine-Hugoniot conditions in this case are

$$
\left[u_{t}\right] d x+\left[c^{2} u_{x}\right] d t=0
$$

where $[f]$ denotes the jump of $f(x, t)$ across $L$. Here $d x / d t=1$ and we get $u_{t}^{+}+a_{2}^{2} u_{x}^{+}=u_{t}^{-}+a_{1}^{2} u_{x}^{-}$. Thus along $L, u$ and two linearly independent directional derivatives are prescribed. In the region $x / t<1$ the weak solution is also a strong solution and is determined by the initial data along the boundary. However, the function $\psi=u_{t}-a_{1} u_{x}$ is constant along the direction $\left(a_{1} d x_{1}, d t\right)$; that is, $\psi_{t}+a_{1} \psi_{x}$ $=0$. Thus the directional derivative $u_{t}^{-}-a_{1} u_{x}^{-}$is uniquely determined on $L^{-}$. We now have three independent directional derivatives determined along $L^{-}$and so the partial derivatives $u_{x}$ and $u_{t}$ are overly determined along $L^{-}$by the initial data. Consequently, a weak solution cannot exist. 
Finally we discuss the question of uniqueness of weak solutions of the second order equation (25). This time we shall assume that

$$
-\sum_{i, j=1}^{n} \frac{a^{i j}(\boldsymbol{x}, t)-a^{i j}\left(\boldsymbol{x}, t^{\prime}\right)}{t-t^{\prime}} v_{i} v_{j} \leqq A^{\prime} \sum_{i=1}^{n} v_{i}^{2}
$$

for some constant $A^{\prime}$ and any numbers $v_{1}, \ldots, v_{n}$. This condition is complementary to the condition (ii) on the quadratic form $a^{i j}$ which was assumed in order to establish existence. Note that both the conditions (ii) and (ii') will be satisfied if we assume that each of the functions $a^{i j}(x, t)$ is Lipschitz continuous in $t$.

We further assume that the coefficient $b$ is Lipschitz continuous in $t$; that $c^{i}$ is Lipschitz continuous in $x_{i}$; and that $b, c^{i}$, and $d$ are bounded on compact subsets of $\mathscr{D}$.

Suppose that there are two distinct weak solutions of (25), say $u_{1}$ and $u_{2}$. For the difference $w=u_{1}-u_{2}$ we get the following integral identity:

$$
\iint_{\mathscr{D}}\left[-w_{t} \phi_{t}+\sum_{i, j=1}^{n} a^{i j} w_{i} \phi_{j}-\phi\left\{b w_{t}+\sum_{i=1}^{n} c^{i} w_{i}+d u+e\right\}\right] d \boldsymbol{x} d t=0
$$

for any test function $\phi$ which vanishes for large $|x|+t$. We shall show that $w=0$ a.e. by showing that

$$
\iint_{\mathscr{D}} F(t, \boldsymbol{x}) w d \boldsymbol{x} d t=0
$$

for any smooth function $F(t, \boldsymbol{x})$ with compact support in $\mathscr{D}$.

Let $F(x, t)$ have support in $0<t<T$ and let $\phi^{k}$ be the solution of the second order equation

$$
\phi_{t t}^{k}-\sum_{i, j=1}^{n}\left(a_{k}^{i j} \phi_{i}^{k}\right)_{j}-b_{k} \phi_{t}^{k}-\sum_{i=1}^{n} c_{k}^{i} \phi_{i}-d_{k}^{\prime} \phi^{k}=F(x, t),
$$

where $d_{k}^{\prime}=d_{k}+\sum_{i=1}^{n} c_{k, i}^{i}+b_{k, t}$.

The solution $\phi^{k}$ is to satisfy the conditions $\phi^{k}(x, T)=\phi_{t}^{k}(x, T)=0$. We seek the solution of the backward hyperbolic problem in $0<t<T$. Set $\psi^{k}(\boldsymbol{x}, t)=\phi^{k}(\boldsymbol{x}, T-t)$; the function $\psi^{k}$ assumes the initial conditions $\psi^{k}(\boldsymbol{x}, 0)=\psi_{t}^{k}(\boldsymbol{x}, 0)=0$ and satisfies the differential equation (30) with the coefficients $a_{k}^{i j}(\boldsymbol{x}, t), b_{k}(\boldsymbol{x}, t), \ldots$ replaced respectively by $a_{k}^{i j}(x, T-t), b_{k}(x, T-t), \ldots$

As in the case of the existence theorem, by the assumptions (ii') we obtain an upper bound on the energy integral

$$
E^{k}(t)=\frac{1}{2} \int_{C(t)}\left(\psi_{t}^{k}\right)^{2}+\sum_{i, j=1}^{n} a_{k}^{i j} \psi_{i}^{k} \psi_{j}^{k} d x
$$

for the function $\psi^{k}(\boldsymbol{x}, t)$-hence also for the function $\phi^{k}(\boldsymbol{x}, t)$. The estimate is uniformly valid as $k \rightarrow \infty$. The region $C(t)$ is the intersection of the hyperplane 
$x_{0}=t$ with an appropriate cone-one of the cones $\Gamma_{\tau}$ which is large enough to contain the support of $F(x, t)$.

Now by the properties of the weak solution $w(x, t)$ we have

$$
\begin{array}{rl}
\iint_{\mathscr{D}} & F(\boldsymbol{x}, t) w(\boldsymbol{x}, t) d \boldsymbol{x} d t \\
\quad=\iint_{\mathscr{D}}\left[w(\boldsymbol{x}, t)\left\{\phi_{t t}^{k}-\sum_{i, j=1}^{n}\left(a_{k}^{i j} \phi_{j}^{k}\right)_{i}+\left(b_{k} \phi^{k}\right)_{t}-\sum_{i=1}^{n}\left(c_{k}^{i} \phi^{k}\right)_{i}-d_{k} \phi^{k}\right\}\right] d \boldsymbol{x} d t \\
\quad=\iint_{\mathscr{D}}\left[-w_{t} \phi_{t}^{k}+\sum_{i, j=1}^{n} a_{k}^{i j} \phi_{j}^{k} w_{i}-b_{k} w_{t} \phi^{k}-\sum_{i=1}^{n} c_{k}^{i} \phi^{k} w_{i}-d_{k} \phi^{k} w\right] d \boldsymbol{x} d t \\
\quad=\iint_{\mathscr{D}}\left[\sum_{i, j=1}^{n}\left(a_{k}^{i j}-a^{i j}\right) \phi_{j}^{k} w_{i}-\left(b_{k}-b\right) \phi^{k} w-\sum_{i=1}^{n}\left(c_{k}^{i}-c^{i}\right) \phi^{k} w_{i}-\left(d_{k}-d\right) \phi_{k} w\right] d \boldsymbol{x} d t .
\end{array}
$$

The smoothed coefficients $a_{k}^{i j}, b_{k}, \ldots$ converge in the $L_{2}$ norm on compact subsets of $\mathscr{D}$ as $k \rightarrow \infty$ to $a^{i j}, b, \ldots$. Furthermore, $a_{k}^{i j}, b_{k}, \ldots$ are uniformly bounded on compact sets as $k \rightarrow \infty$. Also, since the estimate on the energy integral is uniform as $k \rightarrow \infty$ we see that $\phi^{k}$ and $\phi_{i}^{k}$ remain uniformly bounded in the $L_{2}$ norm on compact sets. Letting $k \rightarrow \infty$ and applying Lemma 2 we see that the integral

$$
\iint_{\mathscr{D}} F(x, t) w(x, t) d x d t
$$

is arbitrarily small. The desired result now follows.

\section{BIBLIOGRAPHY}

1. E. D. Conway, Generalized solutions of linear differential equations with discontinuous coefficients and the uniqueness question by multidimensional quasilinear conservation laws, J. Math. Anal. Appl. 18 (1967), 238-251.

2. R. Courant and D. Hilbert, Methods of mathematical physics. Vol. II, Interscience, New York, 1962.

3. I. M. Gelfand, Some questions of analysis and differential equations, Uspehi Mat. Nauk 14 (1959), no. 3 (87), 3-19; Amer. Math. Soc. Transl. (2) 26 (1963), 201-219.

4. N. N. Kuznecov, Hyperbolic systems of linear equations with discontinuous coefficients, Comput. Math. and Math. Phys. 3 (1963), 394-412.

5. O. A. Oleĭnik, Discontinuous solutions of nonlinear differential equations, Uspehi Mat. Nauk 12 (1957), no. 3 (75), 3-73; Amer. Math. Soc. Transl. (2) 26 (1963), 95-172.

UNIVERSITY OF CALIFORNIA, Los Angeles, California 